\title{
Experimental Constraints on Nonequilibrium Interface Kinetic Models
}

\section{Citation}

Aziz, Michael J. 1997. Experimental constraints on nonequilibrium interface kinetic models. Materials Science and Engineering A 226-228: 255.

\section{Published Version}

http://dx.doi.org/10.1016/S0921-5093(97)80040-8

\section{Permanent link}

http://nrs.harvard.edu/urn-3:HUL.InstRepos:2966791

\section{Terms of Use}

This article was downloaded from Harvard University's DASH repository, and is made available under the terms and conditions applicable to Other Posted Material, as set forth at http:// nrs.harvard.edu/urn-3:HUL.InstRepos:dash.current.terms-of-use\#LAA

\section{Share Your Story}

The Harvard community has made this article openly available.

Please share how this access benefits you. Submit a story.

\section{Accessibility}




\title{
Experimental constraints on nonequilibrium interface kinetic models
}

\author{
Michael J. Aziz \\ Division of Engineering and Applied Sciences, Harvard University, Cambridge MA 02138, USA
}

\begin{abstract}
Experimental results relevant to models for nonequilibrium interface kinetics during rapid solidification are reviewed. Models are examined critically in light of these experiments. The kinetic Ising model is shown to compare unfavorably with experiment. The Continuous Growth Model without solute drag and its extension to non-(001) interfaces, the Aperiodic Stepwise Growth Model, account well for all relevant experimental results.
\end{abstract}

Keywords: rapid solidification, undercooling, solute trapping

\section{Introduction}

When rapid solidification occurs, deviations from local interfacial equilibrium become readily apparent. Solute trapping occurs: $k$, the ratio of solute concentration in the solid to that in the liquid at the interface, deviates from its equilibrium value and approaches unity. Kinetic undercooling occurs: the crystal/melt interface becomes undercooled with respect to the equilibrium liquidus temperature for the liquid composition at the interface. The objective of nonequilibrium interface kinetic models is to account for the interface velocity, $v$, and the chemical composition $C_{\mathrm{S}}$ of a growing phase, given the composition $C \mathrm{~L}$ of the parent phase at the interface, the temperature $T$ of the interface, and the interface orientation $\theta$. The models provide "interface response functions" [1] — the "solute trapping function" $k\left(v, C_{\mathrm{L}}, \theta\right)$ and the "velocity-undercooling function" $v\left(T, C_{\mathrm{L}}, \theta\right)$ - which are then used as the interface boundary conditions for the equations of continuum heat and mass transport for rapid solidification processes.

\section{What we need from a model}

The plane-front geometry that can be enforced by pulsed laser melting has permitted the rapid experimental quantification of the interface response functions. Additionally, molecular dynamics simulations provide a different kind of "experimental" data. A successful model should be able to account for the experimental results described below.

(a) The solute trapping function has been measured, over a range of measured interface velocities, for trapping of Bi [2], Sn [3], As [4] and $\mathrm{Ge}[5,6]$ in $\mathrm{Si}$ and for $\mathrm{Sn}$ in $\mathrm{Al}$ [7]. A nonequilibrium partition coefficient has been measured at a single $v$ for $\mathrm{Al}-\mathrm{Cu}$,
Al-Ge and Al-In [7]. To facilitate a more accurate comparison of a wider variety of future models with experiment, the "raw" Si-Bi $k(v)$ data (initial and final concentration vs. depth profiles, measured melt depth vs. time) have now been published [8].

(b) A model must provide reasonable behavior in limiting cases. Enough experimental evidence now exists that models should predict virtually complete solute trapping of dilute $\mathrm{B}$ in $\mathrm{A}$, and virtually complete solute trapping of dilute $\mathrm{A}$ in $\mathrm{B}$, at high velocities for the same set of parameters $[5,9]$.

(c) The velocity-undercooling function for pure $\mathrm{Si}$ (001) has been measured [10-16]. Although some of the measurements are more direct than others, in all cases the reported "interface sluggishness" [17], $\mathrm{d}(\Delta T) / \mathrm{d} v$ or, for the cases under consideration, $\Delta T / v$, near the melting point falls in the range 3$18 \mathrm{~K} /(\mathrm{m} / \mathrm{s})$. A successful model should predict an interface sluggishness in this range with the same parameters used to fit the solute trapping data. For Si (111) the average sluggishness over the range $0-6 \mathrm{~m} / \mathrm{s}$ is approximately $11 \mathrm{~K} /(\mathrm{m} / \mathrm{s})$ [11].

(d) The velocity-undercooling function for alloys has been measured in non-dilute $\mathrm{Si}(\mathrm{As})$. From these experiments, plus the behavior of the measured interface velocity during the solute trapping experiments, it is clear that the interface sluggishness for dilute $\mathrm{Si}$ alloys is virtually identical to that for pure $\mathrm{Si}$. Comparison with the velocity-undercooling function for pure $\mathrm{Si}$ eliminates free parameters and has resulted in the dismissal of many models $[4,18,19]$.

(e) If a model is to be generally valid for solidification - and not merely specific to silicon - it should also be able to match, with a single set of parameters, the solute trapping data for metallic 
alloys [7] while maintaining atomic mobilities high enough to permit solidification velocities of hundreds of meters per second [20].

(f) The measured orientation-dependence of solute trapping in $\mathrm{Si}$ at constant interface velocity displays a sharp peak in the partition coefficient at the (111) orientation and a rapid decrease as the interface orientation is tilted toward (001) or (110) [2123]. A successful model should be able to account not only for the difference between (001) and (111) but also for the singular behavior in the vicinity of (111).

\section{Comparison to models}

We now discuss some kinetic models and compare them to these experimental results.

Jackson et al. [24] have reported interesting new results for simulations of the kinetic Ising model for solute redistribution at the crystal/melt interface during rapid solidification. The model is based on Monte Carlo "jumping" of the crystal/liquid interface across atoms of both solute ("A") and solvent ("B") based only on the energetics determined from the local configurations. "Liquid" atoms are placed on the same lattice as "Solid" atoms and are distinguished from them by a label. A comparison of a simulation for the (01) interface in a two-dimensional square lattice with one early experiment for $\mathrm{Bi}$ trapping in $\mathrm{Si}$ (001) appears to show good agreement. However, the model is in disagreement with other data as discussed below.

Until now, all Ising-based models in which each atom independently makes thermally activated jumps across the interface, biased only by the energetics of its particular initial and final configurations, have been unable to reproduce all of the observed behavior. When the parameters have been adjusted to match solute trapping data under one set of circumstances, other experimental behavior cannot be matched. Typically, for Isingbased models to produce solute trapping in the past, an atomic mobility has had to be reduced so drastically that other unintentional departures from reality are manifested. For example, with the parameters in a Monte Carlo simulation adjusted to permit more trapping on (111) than (001) interfaces [25], the interfacial undercoolings were much greater than indicated by experiment [10-16]. In another example, an analytical model based on similar principles to those of the kinetic Ising model [26,27], with the parameters adjusted to permit trapping of dilute $\mathrm{A}$ in $\mathrm{B}$, did not permit trapping of dilute $\mathrm{B}$ in $\mathrm{A}$ at any speed [5], in conflict with experiments on the $\mathrm{Si}$ Ge system [5,9].

The new simulations of Jackson et al. are a significant step forward in two important ways. For the first time, solute trapping is a consequence of this model using a physically reasonable set of kinetic parameters. Also, for the first time, the kinetic Ising model is rationalized using the paradigm of earlier, successful simple analytical models [28] : "There is a transition between these two extremes [equilibrium partitioning vs. complete solute trapping] when the jump rate for the [solute atoms] is comparable to the rate at which the interface moves. We believe this to be the origin of solute trapping behavior..." [24].

The new simulation appears to be able to match solute trapping behavior at a single interface velocity. However, problems arise when we consider other predictions of the model. The simulation produces a partition coefficient of $k \sim 0.1$ at a reported interfacial undercooling of $\Delta T=285 \mathrm{~K}$. This undercooling is an order of magnitude greater than experimental values. Although $v$ is not reported explicitly by Jackson et al., the experimental information on the undercooling at which a particular partition coefficient is attained comes from combining independent measurements of $k(v)$ and $v(\Delta T)$. The comparison with experiment must therefore be made using $v$ as a "middleman", which is cumbersome; the conclusion, however, is inescapable: the experimental undercooling is far smaller, being only 10-60 $\mathrm{K}$ when $k=0.1$.

In comparing Jackson et al.'s new simulation to Baeri et al.'s experimental depth profile [29] for solute trapping of $\mathrm{Bi}$ in $\mathrm{Si}$ (001), we may first assume that the value of the fundamental time-step of the Monte Carlo simulation can be assigned such that the solidification velocity in the simulation matches Baeri's reported solidification velocity of $v=1.8 \mathrm{~m} / \mathrm{s}$. If this is indeed the case then the reported simulation is very far from matching the measured velocity-undercooling relation for pure Si (001) within the range of experimental uncertainty [10-16]. The reported simulation indicates $\Delta T=285 \mathrm{~K}$ of undercooling at an inferred velocity of $1.8 \mathrm{~m} / \mathrm{s}$, for an interface sluggishness of $\Delta T / v=160 \mathrm{~K} /(\mathrm{m} / \mathrm{s})$. The range of reported experimental values is $3-18 \mathrm{~K} /(\mathrm{m} / \mathrm{s})$ as indicated in (c) above.

The authors compared their simulations to Baeri et al.'s original experiment in which there is considerable uncertainty about the actual solidification velocity. In the early solute trapping studies $[29,30]$ the velocity was estimated using a numerical solution of the heat equation. Later solute trapping experiments on the same $\mathrm{Si}(\mathrm{Bi})$ (001) system were performed with measured velocities [2]. It was also determined and reported [2] that Baeri et al.'s original velocity estimates were in error primarily because the interface velocity was calculated using an assumed value for the thermal conductivity of amorphous silicon that measurements subsequently indicated to be too high. New simulations by Baeri et al. for the original experimental conditions, using more recent data for the thermal conductivity of amorphous silicon, indicate that in the experiment in question $v=2.4 \mathrm{~m} / \mathrm{s}$ if the reported laser pulse energy density was accurate [31]. Baeri's "best-fit" value of the nonequilibrium partition coefficient for this experiment was $k=0.1$; the subsequent experiment [2], in which $v$ was measured instead of estimated, indicates that $k=0.1$ at $v=3.5 \mathrm{~m} / \mathrm{s}$. 
Within this factor-of-two-or-so uncertainty in the velocity associated with Baeri's depth profile, no matter which value of $v$ is chosen for the simulation to match, the reported interfacial undercooling of $\Delta T=285 \mathrm{~K}$ is far too large. Larson et al. $[10,11]$ measured for $v=6 \mathrm{~m} / \mathrm{s}$ an undercooling of at most $140 \mathrm{~K}$ for Si (111) and at most $75 \mathrm{~K}$ for $\mathrm{Si}(001)$, for a maximum interface sluggishness of $\Delta T / v=23 \mathrm{~K} /(\mathrm{m} / \mathrm{s})$ for $(111)$ and $13 \mathrm{~K} /(\mathrm{m} / \mathrm{s})$ for (001). Their best-fit values for the sluggishness were 11.4 $\mathrm{K} /(\mathrm{m} / \mathrm{s})$ for $(111)$ and $5.6 \mathrm{~K} /(\mathrm{m} / \mathrm{s})$ for $(001)$. If the Ising model simulation is associated with an experimental velocity in the vicinity of $2-4 \mathrm{~m} / \mathrm{s}$ (i.e., $1 / 3$ to $2 / 3$ of Larson's $v$ ) then the "experimental" undercooling that the simulation must match should be scaled down accordingly (perhaps not exactly linearly, but not too far therefrom). For example, for an interface velocity of $3 \mathrm{~m} / \mathrm{s}$ the experiment of Larson et al. implies $\Delta T \approx 34 \mathrm{~K}$ for (111) and $\Delta T \approx 17 \mathrm{~K}$ for (001). If instead the undercooling in the simulation is matched to the experimental undercooling then a linear extrapolation would imply a velocity of at least $12.2 \mathrm{~m} / \mathrm{s}$ for (111) and at least $22.8 \mathrm{~m} / \mathrm{s}$ for (001); using instead Larson et $a l$.'s best values of the sluggishness will roughly double these implied velocities. However, the maximum possible solidification velocity is $10 \mathrm{~m} / \mathrm{s}$ for (111) and $15 \mathrm{~m} / \mathrm{s}$ for (001); at higher speeds the growth of the crystal is replaced by growth of an amorphous solid [32-34]. The kinetic Ising model does not account for amorphization because it ignores relevant structural information, keeping all atoms in all phases on a single lattice.

The (01) simple square lattice crystallography of the simulation is actually more appropriately compared to the singular $\mathrm{Si}$ (111) interface than the (001) interface. However, no matter which experimental interface is chosen for comparison, the undercooling in the simulation is far too large. For Si (Bi) (111), $k=0.1$ at $v=0.66 \mathrm{~m} / \mathrm{s}$ [21,22]. With an average interface sluggishness over the range $0-6 \mathrm{~m} / \mathrm{s}$ of $11 \mathrm{~K} /(\mathrm{m} / \mathrm{s})$ [11], the implied interfacial undercooling is $\Delta T \approx 7 \mathrm{~K}$ when $k=0.1$.

The fewer adjustable parameters in the model (or, the more parameters that can be determined by independent means), the better. In the model of Jackson et al., the adjustable parameters appear to be $P_{\mathrm{B}}^{\mathrm{o}} / P_{\mathrm{A}}^{\mathrm{o}}, \Gamma / P_{\mathrm{A}}^{\mathrm{o}}$, the entropies of fusion $\Delta S_{\mathrm{A}}$ and $\Delta S_{\mathrm{B}}$, and the various bond energies $\phi_{\mathrm{AA}}^{\mathrm{LL}}, \phi_{\mathrm{BB}}^{\mathrm{LL}}, \phi_{\mathrm{AB}}^{\mathrm{LL}}, \phi_{\mathrm{AA}}^{\mathrm{CL}}, \phi_{\mathrm{BB}}^{\mathrm{CL}}, \phi_{\mathrm{AB}}^{\mathrm{CL}}$, $\phi_{\mathrm{AB}}^{\mathrm{LC}}, \phi_{\mathrm{AA}}^{\mathrm{CC}}, \phi_{\mathrm{BB}}^{\mathrm{CC}}, \phi_{\mathrm{AB}}^{\mathrm{CC}}$, where "A" indicates "Si", "B" indicates "Bi", "C" indicates "Crystal" and "L" indicates "Liquid". Certainly not all of these parameters are independently adjustable: the melting point and entropy of fusion of pure $\mathrm{Si}$ and the equilibrium partition coefficient provide three constraints. Matching the measured liquid-phase diffusivity provides a fourth constraint among these 14 parameters, resulting in 10 adjustable parameters. Reasonable choices were made for many of these adjustable parameters; however, the choices are not unique and therefore they remain adjustable parameters.
The excessive undercooling predicted by the kinetic Ising model is not unique to singular orientations. In a subsequent publication, [35], the kinetic Ising model's interface response functions were examined for $\mathrm{Si}-\mathrm{Bi}$ on both the (10) and (11) interfaces of a two-dimensional square lattice, and comparison was made to the (111) and (001) interfaces, respectively, of the three-dimensional experimental system. However, the comparison with experiment was incomplete. Below are presented other comparisons of the (11)-simulated vs. (001)experimental interface, for which the most experimental data are available:

From Fig. 6 of Jackson et al. [35], the results of the simulations are plotted as $k$ vs. $\beta$, where

$$
\beta \equiv v^{2} /\left(a \Gamma v^{+}\right)
$$

here $a$ is the interatomic spacing $(2.35 \AA$ for $\mathrm{Si}), v^{+}$is the "arrival rate" (or the maximum growth rate at infinite driving force) and $\Gamma$ is the jump rate in the bulk of the liquid, which is related to the diffusion coefficient in the liquid, $D$, by

$$
D=a^{2} \Gamma / 4
$$

$D=2 \times 10^{-4} \mathrm{~cm}^{2} / \mathrm{s}$ for $\mathrm{Bi}$ in $\mathrm{Si}[2,23,29,30]$. Fig. 6 of Jackson et al. examines for the (11) interface the cases $g=20$ and $g=2$, where the adjustable parameter $g$ is defined by

$$
g \equiv \Gamma a / v^{+}
$$

(The introduction of $g$ is necessary to address a dimensional inconsistency in Jackson et al.'s definition of $\Gamma$ ). Combining (2) and (3), we have

$$
D=g v^{+} a / 4
$$

The authors chose to fit an experimental interface sluggishness for $\mathrm{Si}(001)$ of $\Delta T / v=8.33 \mathrm{~K} /(\mathrm{m} / \mathrm{s})$, resulting in $v^{+}=75 \mathrm{~m} / \mathrm{s}$ for the (11) interface. This can be used to fix the value of $g$ using (4), resulting in $g=4.54$. This value falls between the two simulation values presented in Fig. 6 of Jackson et al., so we can presume that results for $g=4.54$ would lie along the dashed curve in that figure. From this curve, $k=0.1$ at $\beta=2 \times 10^{-2}$, where, using (1) and (3),

$$
\beta=\left(v / v^{+}\right)^{2} / g
$$

Hence, from (5), $k=0.1$ at

$$
v=v^{+}(g \beta)^{1 / 2}
$$

$$
=23 \mathrm{~m} / \mathrm{s}
$$


for the (11) interface simulations. The experimental interface sluggishness of $\Delta T / v=8.33 \mathrm{~K} /(\mathrm{m} / \mathrm{s})$ to which the simulations were fit then implies an undercooling of $\Delta T=192 \mathrm{~K}$ in the simulations. Experimental results for $\mathrm{Bi}$ in $\mathrm{Si}(001)$ are $k=0.1$ at $v=3.5 \mathrm{~m} / \mathrm{s}$ [2] and, using the same quoted interface sluggishness, $\Delta T=29 \mathrm{~K}$. Thus the simulated velocities and undercoolings for the (11) interface are far too large to fit the (001) solute trapping experiment. Additionally, with

$$
\beta=v^{2} a /\left(4 D v^{+}\right)
$$

from (1) and (2), we obtain $\beta / v^{2}=3.9 \times 10^{-5}(\mathrm{~m} / \mathrm{s})^{2}$ rather than the value of $5 \times 10^{-3}(\mathrm{~m} / \mathrm{s})^{2}$ reported by Jackson et al. as being used to compare the simulations to experiment in their Fig. 7. No matter which value of $\beta / v^{2}$ is used, however, note that although the slope of the curve in Fig. 7 matches that of the early solute trapping data rather well, the curve rises too steeply to match the slope of the later, more accurate data for which the velocity was measured rather than estimated using heat-flow simulations.

The equilibrium Ising model is a powerful tool for investigating the equilibrium long-wavelength fluctuations of an interface near its critical temperature. Near the critical point, so-called "universality" properties are exhibited which are independent of crystallographic details and of the specific nature of the shortrange interaction between sites. How, then, can there be difficulty in fitting rapid solidification experiments with the kinetic Ising model? It is important to keep in mind the highly nonequilibrium nature of the rapid solidification process, which therefore is not necessarily subject to universality properties. Additionally, the interatomic interactions responsible for solute trapping are of a highly localized nature (e.g., nearest-neighbor atomic configurations at moving step edges), and highly local information is not subject to universality behavior even in equilibrium. Hence highly localized structural information can be important, and the kinetic Ising model is not designed to accurately represent local structural information at the crystal/melt interface.

The main physical shortcoming of the kinetic Ising model applied to highly local details of the crystal/melt interface is that for a real-world solute atom to crystallize, it never has to make a thermally-activated "jump" from the liquid into the crystal. Rather, a solute atom can do nothing and still find itself on a lattice site if all of its solvent neighbors have crystallized. In the kinetic Ising model, a recalcitrant solute atom may never crystallize even when all of its neighbors are crystalline! In fact, in reporting the results of such a model one should be careful to distinguish between the unphysical process of "liquid" B atoms being "trapped" by becoming surrounded by "solid" A atoms while remaining liquid, and the more physically reasonable process of "liquid" B atoms being induced to make a transition to "solid" B while at the interface. The difference between the local structure in the liquid and the solid is missing from the Ising model. The mean free path in the liquid tends to be much smaller than the nearest-neighbor distance in the lattice. There is no mechanism in the model for accounting for this difference when atomic interchanges are made between adjacent lattice sites. For these and other reasons the Ising model is an imperfect model for the crystal/melt interface, other than for large-scale fluctuations at the critical temperature.

The critical temperature, at which certain universality behavior should be observed, is not to be confused with the equilibrium melting temperature. One indication that the kinetic Ising model is not totally satisfactory even for the crystal/melt interface in equilibrium is that it cannot match experimental behavior for silicon without treating the entropy of fusion as an adjustable parameter. Clearly the nature of short-range interatomic interactions are indeed important in this case. Recently, some progress may have been made in improving the fit to measured velocities and undercoolings by using a three-dimensional diamond cubic lattice [36]; however, successful fitting still appears to require adjustments to the entropy of fusion.

The kinetic Ising model should faithfully represent experiment if all structural details are unimportant. It is therefore of interest to see how closely the model can reproduce experiment; the fewer free parameters, the better. The most difficult challenge for the model is likely to be in metallic systems, discussed in (e) above. In metals, solute trapping occurs at relatively slow speeds (meters per second) [7] whereas significant undercooling does not occur until a significant fraction of the speed of sound (kilometers per second) [20]. These experiments indicate that the mobility for solute-solvent redistribution across the interface and that for interface motion may differ by up to three orders of magnitude. In the model there is no distinction between the two, and reproducing both phenomena with a single set of parameters should be difficult.

These and other problems with the kinetic Ising model have been evident for some time. I therefore proposed a model not based on independent hopping of atomic species across an interface, but rather on a particular form of coupled transport across the interface $[17,22,37,38]$. Such a treatment permits widely differing mobilities for solute-solvent redistribution and interface motion. Rather than using the reference frame of individual atomic jumps across the interface, the Continuous Growth Model assumes that a single simple rate equation can be written down for solute-solvent redistribution across the interface and that a separate simple rate equation can be written down for alloy crystallization,

$$
v\left(T, C_{\mathrm{S}}, C_{\mathrm{L}}\right)=A[1-\exp (\Delta G / R T)],
$$

where $\Delta \mathrm{G}$ is a measure of the driving force for solidification, and the parameter $A$ is determined by the pure-solvent $v(T)$ relation. Experiment has shown $[4,18,19]$ that the version of the Continuous Growth Model without solute drag, in which $\Delta G=$ 
$C_{\mathrm{S}} \Delta \mu_{\mathrm{B}}+\left(1-C_{\mathrm{S}}\right) \Delta \mu_{\mathrm{A}}$ is the Gibbs free energy change per mole of alloy solidified $(\Delta G<0)$, fits the data and that no solute drag model fits all of the data. Admittedly, despite excellent agreement with experiment [4,18], equation (9) is phenomenological and is not based on a detailed atomistic [39] mechanism. A successful mechanistic model therefore remains highly desirable. It should, however, be kept in mind that there are many other phenomenological models that have proven very useful without being based on a detailed atomistic mechanism [40-46]. In general it might be anticipated that the successful phenomenological model might be used as guidance in the development of a successful mechanistic model. It would be interesting to compare the predictions of the kinetic Ising model to equation (9), in order to see whether for any realistic solidification process an experiment might be devised that could distinguish one from the other. Because of all the parameters in the kinetic Ising model, this must be done with the utmost care. The ultimate test must remain compatibility with experiment.

\section{Conclusions}

There is now a large body of experimental data which nonequilibrium interface kinetic models must reproduce in order to become established. At present, neither the new kinetic Ising model of Jackson et al., nor any previous incarnation of the kinetic Ising model, has been demonstrated unambiguously to match any of (a)-(f). The Continuous Growth Model and its extension to non-(001) interfaces, the Aperiodic Stepwise Growth Model [22], has been demonstrated to account successfully for (a)-(e) with only two free parameters for each alloy system, and for the orientation-dependence (f) with a third free parameter.

\section{Acknowledgments}

The author acknowledges valuable discussions with K.A. Jackson and P. Baeri. This work was supported by NSF-DMR92-08931.

\section{References}

1J.C. Baker and J.W. Cahn in Solidification, (Am. Soc. Metals, Metals Park, Ohio, 1970), p. 23.

2M.J. Aziz, J.Y. Tsao, M.O. Thompson, P.S. Peercy and C.W. White, Phys. Rev. Lett. 56, 2489 (1986).

${ }^{3}$ D.E. Hoglund, M.J. Aziz, S.R. Stiffler, M.O. Thompson, J.Y. Tsao and P.S. Peercy, J. Crystal Growth 109, 107 (1991).

${ }^{4}$ J.A. Kittl, M.J. Aziz, D.P. Brunco and M.O. Thompson, J. Cryst. Growth 148, 172 (1995).

${ }^{5}$ M.J. Aziz, J.Y. Tsao, M.O. Thompson, P.S. Peercy, C.W. White and W.H. Christie, Mater. Res. Soc. Symp. Proc. 35, 153 (1985).

6D.P. Brunco, M.O. Thompson, D.E. Hoglund, M.J. Aziz and H.J. Gossmann, J. Appl. Phys. 78, 2575 (1995).

7P.M. Smith and M.J. Aziz, Acta Metall. Mater. 42, 3515 (1994).
8M.J. Aziz, Metall. Mater. Trans. A 27, 671 (1996).

${ }^{9}$ Q. Yu, M.O. Thompson and P. Clancy, Phys. Rev. B 53, 8386 (1996).

${ }^{10}$ B.C. Larson, J.Z. Tischler and D.M. Mills, J. Mater. Res. 1, 144 (1986).

${ }^{11}$ B.C. Larson, J.Z. Tischler and D.M. Mills, Mater. Res. Soc. Symp. Proc. 100, 513 (1988).

${ }^{12}$ M.O. Thompson, P.H. Bucksbaum and J. Bokor, Mater. Res. Soc. Symp. Proc. 35, 181 (1985).

${ }^{13}$ D.H. Lowndes, J. G.E. Jellison, S.J. Pennycook, S.P. Withrow and D.N. Mashburn, Appl. Phys. Lett. 48, 1389 (1986).

${ }^{14}$ G.J. Galvin, J.W. Mayer and P.S. Peercy, Appl. Phys. Lett. 46, 644 (1985).

15P.H. Bucksbaum and J. Bokor, Phys. Rev. Lett. 53, 182 (1984).

16J.Y. Tsao, M.J. Aziz, M.O. Thompson and P.S. Peercy, Phys. Rev. Lett. 56, 2712 (1986).

${ }^{17}$ M.J. Aziz and W.J. Boettinger, Acta Metall. Mater. 42, 527 (1994).

18J.A. Kittl, M.J. Aziz, D.P. Brunco and M.O. Thompson, Appl. Phys. Lett 64, 2359 (1994).

19 J.A. Kittl, M.J. Aziz, D.P. Brunco and M.O. Thompson, Mater. Res. Soc. Symp. Proc. 398, 119 (1996).

${ }^{20}$ C.A. MacDonald, A.M. Malvezzi and F. Spaepen, J. Appl. Phys. 65, 129 (1989).

21M.J. Aziz and C.W. White, Phys. Rev. Lett. 57, 2675 (1986).

22L.M. Goldman and M.J. Aziz, J. Mater. Res. 2, 524 (1987).

${ }^{23}$ R. Reitano, P.M. Smith and M.J. Aziz, J. Appl. Phys. 76, 1518 (1994).

${ }^{24}$ K.A. Jackson, G.H. Gilmer and D.E. Temkin, Phys. Rev. Lett. 75, 2530 (1995).

25G.H. Gilmer, Mat. Sci. Eng. 65, 15 (1984).

26K.A. Jackson, in Surface Modification and Alloying by Laser, Ion and Electron Beams, edited by J.M. Poate, G. Foti and D.C. Jacobson (Plenum Press, New York, 1983), p. 51.

${ }^{27}$ K.A. Jackson, G.H. Gilmer and H.J. Leamy in Laser and Electron Processing of Materials, edited by C.W. White and P.S. Peercy (Academic Press, New York, 1980), p. 104.

${ }^{28}$ M.J. Aziz, Appl. Phys. Lett. 43, 552 (1983).

${ }^{29}$ P. Baeri, G. Foti, J.M. Poate, S.U. Campisano and A.G. Cullis, Appl. Phys. Lett. 38, 800 (1981).

${ }^{30}$ C.W. White, B.R. Appleton and S.R. Wilson, in Laser Annealing of Semiconductors, edited by J.M. Poate and J.W. Mayer (Academic, New York, 1982).

31P. Baeri and R. Reitano, private communication (1995).

${ }^{32}$ M.O. Thompson, J.W. Mayer, A.G. Cullis, H.C. Webber, N.G. Chew, J.M. Poate and D.C. Jacobson, Phys. Rev. Lett. 50, 896 (1983).

33J.A. Yater and M.O. Thompson, Mater. Res. Soc. Symp. Proc. 100, 93 (1992).

34J.A. Yater and M.O. Thompson, Phys. Rev. Lett. 63, 2088 (1989).

${ }^{35}$ K.A. Jackson, G.H. Gilmer, D.E. Temkin and K.M. Beatty, J. Cryst. Growth 163, 461 (1996).

36K.A. Jackson, private communication (1996).

37M.J. Aziz, J. Appl. Phys. 53, 1158 (1982). 
38M.J. Aziz and T. Kaplan, Acta Metall. 36, 2335 (1988).

39I have taken care to distinguish between the terms "atomistic mechanism" and "physical mechanism". An example of an unphysical atomistic mechanism is given three paragraphs previously.

${ }^{40}$ W.A. Tiller, K.A. Jackson, J.W. Rutter and B. Chalmers, Acta Metall. 1, 428 (1953).

41W.W. Mullins, J. Appl. Phys. 28, 333 (1957).

42W.W. Mullins, J. Appl. Phys. 30, 77 (1959).

${ }^{43}$ C. Herring, in Structure an Properties of Solid Surfaces, edited by R. Gomer and C.S. Smith (University of Chicago Press, Chicago, 1953), p. 5.

${ }^{44}$ W.W. Mullins and R.F. Sekerka, J. Appl. Phys. 35, 444 (1964).

${ }^{45}$ S.M. Allen and J.W. Cahn, Acta Metall. 27, 1085 (1979).

46 M. Kardar, G. Parisi and Y. Zhang, Phys. Rev. Lett. 56, 889 (1986). 